\title{
II. Ueber zeitweises Fehlen der Cylinder im Urin bei Nephritis.
}

\author{
Von Dr. med. Ernst Sehrwald,
}

Privatdocent an der Universität Jena.

Voll verschiedenen Autoren wird die Thatsache erwälınt, dass sie trot\% wiederholter und sorgfältiger Untersuchung niclit imstande waren, in manchen Urinen Cylinder aufyufinden, obgleich ansgeprägte Albuminurie bestand, und der Urin sogar ein reichliches Sediment lieferte. Diese Erscheinung muss umsomehr anffallen, da eime Reihe von Forschern geradezu erklärt, dass das Auftreten von Albuminurie und von Cylindern zwei so eng verschwisterte Processe sind, dass ihr gemeinsames Vorkommen fast als Gesetz gelten muss.

Bartels ${ }^{1}$ ), welcher dieser Anschauung durchaus huldigt, salı sich trotzdem gezwungen, vereinzelte Ausnalımen von diesem Geset\% zu constatiren, ohne fïr dieselben allerdings eine ausreichende Erklärung geben zu können. Auch Bizzozero ${ }^{2}$ ) u. a. erwähnen dies Fehlen der Cylinder bei typischer Albuminurie als etwas sehr auffallendes.

Mir selbst sind schon früher wiederholt Fälle der Art vorgekommen, ein besonders markanter aber wieder in cler letzten Zeit. Hier bestand bei einem jungen Mädcheu seit 2-3 Monaten eine ziemlich hochgradige, chronische, parenchymatöse Nephritis mit allen typischen Symptomen, starker Albuminnie, Oedemen inl Gesicht und an den Extremitäten u. s. w. Obwohl nmm das Krankheitsbild dauernd ein äusserst gleichmässiges Verlıalten zeigte, so ergab doch die mikroskopische Untersuchung des Urinsedinıents an manchen Tagen nicht die Spur eines Cyliuders und zu anderen Zeiten bei genau der gleicheu Metlıode äusserst zahlreiche Cylinder der verschiedensten Grösse und Art, unter denen die Epithel- und Köruchen cylinder weitaus prïvalirten, so dass an der Richtigkeit der Diagnose kein Zweifel sein konnte.

Für diese höchst anffallende Erscheiunng hat bisher nur Bizzozero eine Erklärung zu geben versucht. Er nimmt au, dass mit dem Auftreten des Eiweisses wohl auch riemlich gleichzeitig die Cylinderbildung beginıt. Wührend aber der eiweisshaltige Uriu sofort und ohue Schwierigkeit aus der Niere abfliessen und die Albuminurie dem Nachweis \%ugänglich machen kaun, haften die Cylinder in der Niere fester und brauchen längere Zeit, bis sie abgestossen werden.

Wenn diese Erklärung überhaupt richtig ist, so kann sie doch nur fïr einzelne Fälle genügen und für diese wiederuın nur beim Beginn der Affection. Während der ersten Tage ist es bei einer Albuminurie wohl denkbar, dass die Cylinder noch nicht mit herausgeschwemmt werden, sobald aber dann die Abstossung der $\mathrm{Cy}-$ linder einmal begonnen hat und die Albuminurie gleichmässig andauert, liegt bei Zugrmudelegung dieser Erklärung überhaupt keine Möglichkeit mehr vor, dass die Cylinder in dem eiweisshaltigen Urin fellen sollten. Allerdings ist ja festzuhalten, dass eine eiweisshaltige Urinportion, deren Eiweiss zum Theil zur Cylinderbildung verwendet wird, meist viel früher ausgeschieden wird, als die aus ihr abgeschiedenen Cylinder. Das Eiweiss im entleerten Urin ist also fast immer vor viel kïrzerer Zeit von der Niere geliefert worden als die Substanz der gleichzeitig mit austretenden

1) v. Ziemssen, Handb. d. speciellen Pathol. u. Therapie Bd. 9, 1875. - C. Bartels, Krankheiten des Harnapparates p. 78, 79

i) G. Bizzozero, Handbuch d. klin. Mikroskopie 1883, p. 205 u. f. 
Cylinder, und das spricht sich ja anch deutlich in den hänfigen -Altersveränderungen" aus, welche die Cylinder zeigen. Sobald aber danu überhanpt die Abstossung der Cylinder begonuen hat, ist keine Möglichkeit mehr abzusehen, weshalb sie bei fortdanernder Albuminurie plötzlich einmal fehlen sollten.

Ueberdies scheinen gar nicht alle Cylinder in der Niere anfangs so festgehalten zn werden, wie dies Bizzozero meint. Wenigstens treten die hyalinen Cylinder nach Beobachtungen von Bartels zuweilen fast im selben Moment im Urin auf, wie das Eiweiss. So fanden sich bei einem völlig gesunden Maıne, der einen heftigen Stoss anf den Rïcken erlitt, schon in der ersten nach 5 Stunden entleerten Harnportion hyaliue Cylinder neben Eiweiss, und in einem zweiten Fall, wo die Albuminurie artificiell durch eine Lammblnttransfusion erzengt war, waren hyaline Cylinder schon 2 Stundeu nach der Operation zugleich mit dem Eiwciss nachweisbar. Also auch diese Thatsachen widersprechen der Annalıme von Bizzozero.

Es erwächst daher die Anfgabe, nach weiteren Momenten zu snchen, welche für unsere Fälle eine Dentung ermöglichen. Um die hierbei thätigen Factoren zn findeu, enpfiehlt es sich, ïberlanpt erst einmal genauer festznstellen, zn welchen Zeiten denu in den betreffenden Urinen die Cylinder fehlen, und wanu sie auftreten, und zu sehen, ob irgend eine Gesetzmässigkeit in diesen Sclıwankungeu nachweisbar ist.

Untersucht iuan die Gesammturiumengen der einzelneu Tage, so ist vou irgend einer Regel iru Vorkommen der Cylinder entfernt nicht die Rede. Ich war daher genöthigt, einen anderen Weg einznschlagen und zunächst zu fragen, ob nicht die einzelnen Haruportionen, die während eines Tages eutleert werden, ähnliche Differenzen in der Zahl der Cylinder anfweisen, wie die Urine verschiedener Tage.

Ich liess zu dem Zweck an verschiedeneu Tageu den Harn stiindlich entleeren und anffangen, andere Male dann aber auch in längeren, etwa 3 stïndigen Pansen. Die Urine wurden sowohl frisch, als anch nach 24stündigem Sedimentiren anf die Meuge ihrer Cyliuder untersucht. Während in allen Urinportiouen eiues Tages die Eiweissmengen bedeutende und fast gleich hohe waren, zeigte die Zahl der Cylinder ganz bedeutende Schwaukungen.

Anf der ersteu Curve z. B., die ich zur Erlänternng beifüge, enthielt der Uriu früh um $6 \mathrm{Uhr}^{\circ}$ sehr zahlreiche Cylinder, um

Ghronische parenchymatöse Nephritis.

\section{Stündliche Entleerung des Harns.}

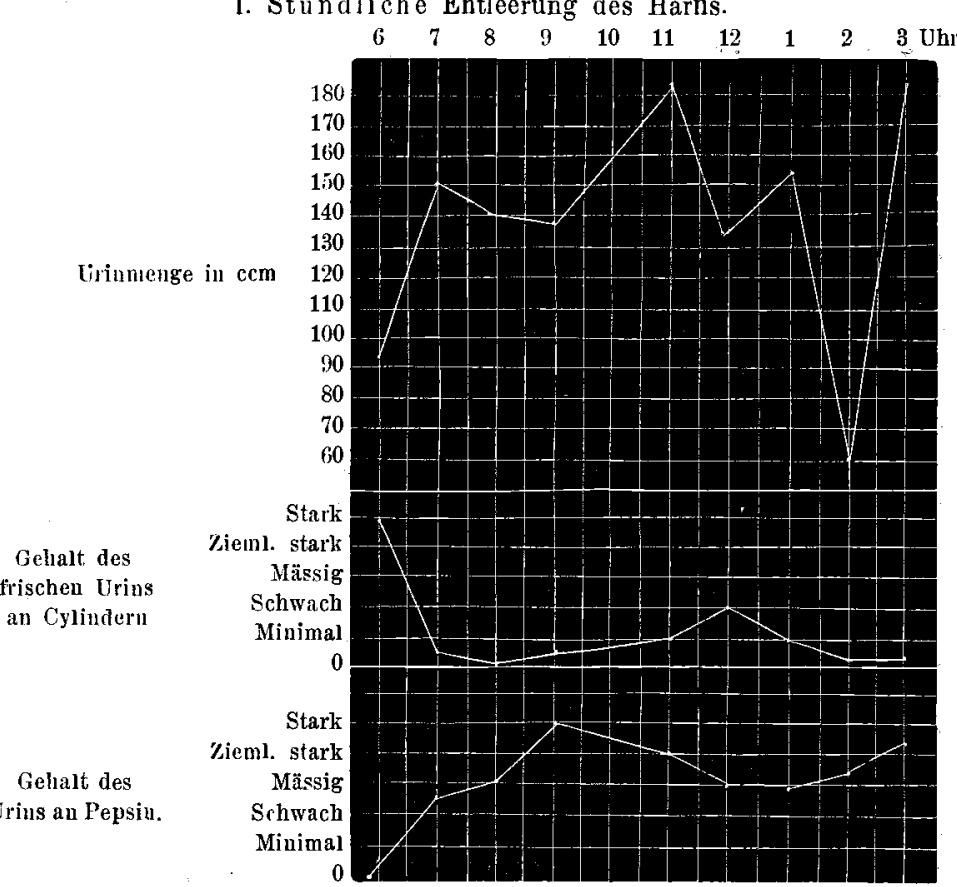

7 Uhr sank die Zahl ganz bedentend ab, und um 8 Uhr war überhanpt kein Cylinder mehr uachweishar. Danu stieg die Zahl derselben allmählich nud gleichmässig wieder an bis $\mathbf{1 2} \mathrm{Uhr}$, erreichte aber nur eíne gerínge Höhe, um dann ziemlich schuell wieder fast bis anf Null zu fallen. Nachdem der Urin 24 Stunden sedimentirt hatte, ergab auch jetzt die Untersuchung fast durchans dieselbeu relativen Mengeuverhältuisse.

Die zweite Curve illustrirt eiuen Tag, an welchem der Urin in grösseren, 2-3 stündigen Pauseu anfgefangen war. Hier ist die Zahl der Cylinder während der ganzen ersten Hälfte des Tages bis 1 Uhr eine sehr minimale und allmählich noch abuehmende, nach 1 Uhr steigt aber dann die Zahl plötzlich gauz bedeutend an.
I1. Möglichst seltene Entleerung des Urins.

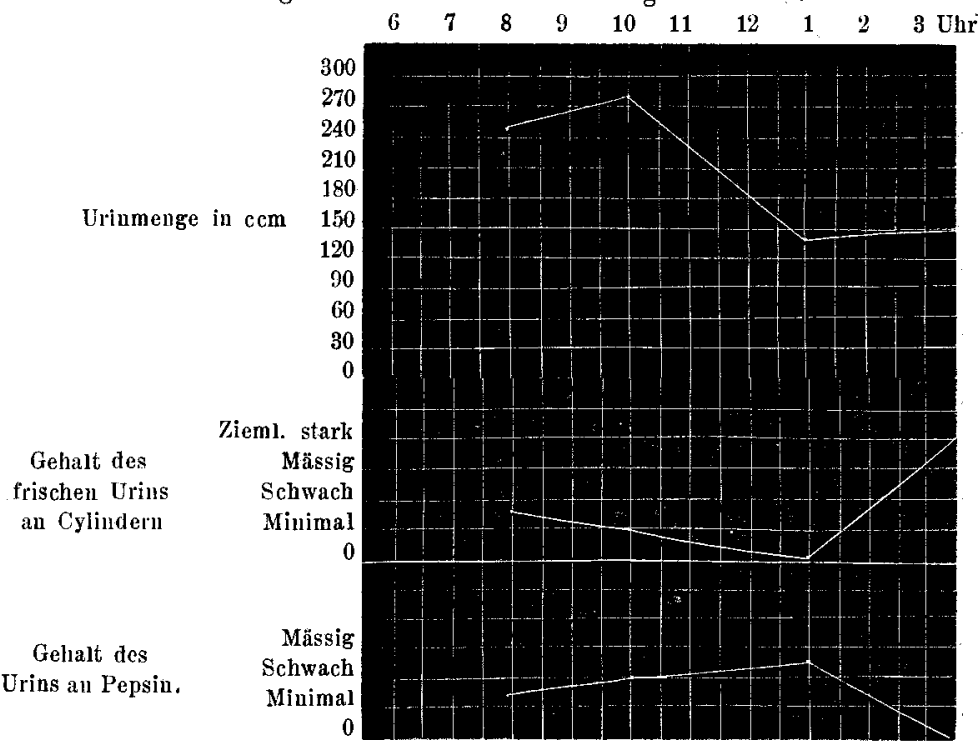

Wir sehen also, dass die Zahl der Cyliuder uicht bloss.täglich, sondern sogar stïudlich ganz bedentendeu Schwankungen unterliegen kann, nnd müsseu nach einem Factor snchen, der eine stündliche iudifferente Nierenarbeit zn erklären vermag. Die Bizzozero'sche Hypothese ist selbstverständlich dieser Thatsache gegeniiber völlig unhaltbar.

Man könnte unn zuuächst darau denken, dass vielleicht die Gesammtarbeit der Niereu zu den einzelueu Tagesstundeu eine quautitativ sehr verschiedeue sei, dass zn Zeiten eiu bedentender Harustrom geliefert wird, der grosse Massen Geröll aus der Niere mitzuführen vermag, nud dass andere Male eine spärliche Urinmenge anch nur wenige Cylinder mitreisst.

Gegen diese Auffassung sprechen sofort die stündlich entleerten Uriumengen, die in durchaus keiner Parallele mit der Menge de Cylinder stehen. Ausserdem wäre es nach dieser Erklärung anch völlig unmöglich, dass grössere Harnportionen einmal ganz frei vou Cylindern gefunden würden, wie es ja vorkommt.

Man wird daher anf eine andere, viel plausiblere Deutung geführt, welche uns durch die Thatsachen der Physiologie an die Haud gegeben wird. Nach den schönen Untersuchnngen von Heideuhain 1) ist in einem bestimmten Moment niemals eine ganze Niere auf eiumal in Thätigkeit. Es arbeiten vielmehr immer un bestimmte Abschnitte der Niere, die daun von anderen inzwischen ausgeruhten abgelöst werden. Bei der erkrankten Niere wird man ein gauz ähnliches Alterniren der eiuzelnen Abschuitte annehmen müssen.

Da nnn, wie die anatomische Untersnchnng zeigt, bei den meisten Nephritiden in der Regel nicht die ganze Niere diffus gleich stark erkranlst, sonderu manche Bezirke stärker, andere schwäclıer afficirt sind, so könnte es leicht gescheheu, dass die Hauptarbeit \%u einer bestimmten Stunde von einem mehr normalen Abschnitt geleistet würde, der dann einen Urin mit wenimen oder selbst gar keinen Cylindern liefern müsste, während $z u$ einer anderen Stutude ein in höherem Grade erkrankter Theil secernirt und in seinem Secret zahlreiche Cylinder mitfiihrt.

Der Beweis fiir die Richtigkeit oder Unhaltbarkeit dieser Auffassuug ist leicht zul führen. Ist sie richtig, so muss anch in del anderen Eigenschaften des Urins diese abwechselnde Arbeit gesunder nud erkrankter Partieen sich anssprechen. Zur Zeit, wo ein gesunder Abschnitt secernirt, muss die Harumenge eine grössere sein, und es muss der normalere Uriu, wie er weniger Cylinder führt, auch wéniger Eiweiss enthalten, event. beim Fehlen der Cylinder fast eiweissfrei sein. Umgekehrt müsste während der Thïtigkeit kranker Bezirke wenig Urin mit viel Eiweiss nnd zahlreichen Cylindern producirt werden. Beides ist nun aber durchans nicht der Fall.

Ich habe schon erwähnt, dass die einzelnen Urinportionen, unbekümmert um die Zahl der Cylinder, alle starken und aunäherud gleich starkeu Eiweissgehalt anfwiesen, dass also die Mengen von Eiweiss und Cylindern durchans keine Parallele aufweisen. Fermer lehrt aber anch ein Blick anf die Harumengen, dass die grőssten Urinquantitäten durchans nicht mit den niedrigsten Zahleu für die Cyliuder zusammenfalleu.

Diese Hypothese vermag also ebensowenig wie die erste den Thatsachen gerecht zu werden nnd ist daher gleichfalls abznweisen. Allerdings soll damit nicht gesagt sein, dass diese alteruirende Arbeit nicht doch in nutergeorduetem Maasse zu der Erscheinung

1) R. Beidenhain, Hermann's Handb. d. Physiol. V, 1. Th. 
mit beiträgt. Die Hauptpunkte ist sie jedenfalls nicht instande zu erklären.

Ehe man sich in weiteren Hypothesen versucht, wird es gut sein, zu fragen, ob sich nicht einige Thatsachen noch auffinden lassen, welche die Basis einer Hypothese etwas breiter und sicherer zu fundamentiven imstancle wären.

In der That ergiebt die mikroskopische Untersuchung noch einige bemerkenswerthe 'Thatsachen. Vor allem weisen die Cylincler tagsïber nicht nur hochgradige quantitative, sondern auch qualitative Differenzen auf. Diese verschiedene Beschaffenheit prïgt sich am deutlichsten an den livalinen Cylindern und an den Epithelund Körnchencylindern aus, soweit diese eine hyaline Grundlage besitzen. Sind zu einer Stunde grosse Mengen Cylinder in Urim vorhanden, so sind diese Cylinder auch durchgehends gut ausgebildet, verhältnissmüssig lang, und die lyalinen Partieen scharf contourirt und gut erkennbar. Sind $\%$ an anderen Zeiten nur wenige Cylincler im Urin nacliweisbar, so ist ihre Grundsubstallz viel zarter nnd oft inur schwierig erkennbar. Vielfach gelingt es überhaupt ıur bei Beachitung der eigenthümlichen, streifenförmigen Anordnung von Körnchen und Zellen, die verbindende Kittsubstan\% noch \%u entdecken. Zugleich prävaliren jetzt kur\%e Cylinder und kleinere 'Trïmmer. Neben diesen melr schemenhaft gewordenen Cylindern sind im Präparat jetzt zahlreiche freie Körtuchenhanfen, Zellkerne u. s. w. zu finden. Fehlen endlich die Cylinder im Urin ganz, so ist damit durchaus nicht gesagt, dass anch sonstige corpusculäre Elemente in ihm nicht enthalten wären. Meist ergaben diese Urine sogar eiı ziemlich beträchtliches Sediment, dessen Formelemente bei sonst intacten Harnwegen selbstverständlich nur von der Niere geliefert sein konnten. Dies Sediment bestand vorwiegend ans Körnchenhaufen, besouders feinsten Fetttröpfchen. Diese Häufchen waren manchmal als kurze Cylinder angeordet, ohne dass sich aber eine Zwischensubstan\% hätte nachweisen lassen. Meist lagen sie isolirt oder hatten sich zu unregelmässigen Gruppen zusammengeballt. Als weiteres Formelement fallen freie Zellkerne noch darin auf.

Vergleicht man diese Befunde zu verschiedenen Zeiten, so hat man erst zahlreiche, gut ausgeprägte Cylinder, diese werden dann warter und blasser, mehr schemenhaft, verschwinden zum Theil, und statt ihrer sind dann nur Körnchenhanfen vorhanden. Eudlich sind überhanpt alle Cylinder als solche verschwunden und durch selır zahlreiche, manchmal noch in Cylinderform angeordnete Haufen von Detrituskörnchen ersetzt.

Diese verschiedenen Bilder zwingen geradezu zu der Annahme, dass man in alleu Harnportionen ursprïnglich reichlich Cylinder hat, die aber yu gewissen Zeiten der Aufösung verfallen und dadurch erst zarter werclen und in Stücke zerbröckelı, und sich endlich ganz auflösen und nur die unlöslichen Körnchen nnd Kerne zurücklassen. Wir würden also aunehmen dürfell, dass die einzelnen Haruportionen von der Niere urspriinglich nicht nur annähernd gleich grosse Mengen von Eiweiss, sondern anch von Cylindern geliefert erhalten, dass $z u$ manchen Zeiten die Cylinder in Urin aber leichit und ausgiebig zerstört werden.

Es ist nur die Frage, wolurch eine Wiederauflösurig der $\mathrm{Cy}$ lincler im Urin bewirkt werlén kann. Die chemischen Stoffe, dureh welche wir kïnstlich eine Lösung der Cylincler herbeizufiihreu vermögen, können nicht in Betracht kommen. So lösen sich hyaline Cylinder \%. B. in starker Essigsäure auf; ferner nach den Untersuchungen Rovida's ${ }^{1}$ ) in Mineralsäuren, \%. B. $1 / 3,1 / 2-2 / 3$ concentrinter Salpetersäure. Süuren von dieser Concentration - sind aber im Urin niemals vorhanden. Ebenso ist die Auflösung der Cylinder beim Erwärmen des Urins auf $65-80^{\circ} \mathrm{C}$ oder beim Erwärmen der Cylinder in destillirtem Wasser auf $20-40^{\circ} \%$ fur Frklärung der angeführten Thatsachen nicht verwentbar.

Da die einfache chemische Lösung nicht in Betracht kommit, ist zo untersuchen, ob nicht ein mehr vitaler Lösungsprocess durch geformte oder ungeformte Fermente hier eine Rolle spielt.

Geformte Fermente, Mikroorganismen, vermögen die Cylinder ansgiebig zu zerstören. Lässt man cylinderlıaltigen Urin längere Keit an der Luft stehen, so finden sich die zahlreich auftretenden Mikroorganismen zunächst nur in der Urinttüssigkeit, bald aber dringen sie auch in die Cylinder ein und durchsetzen diese so rejchlich, dass man sie mit Recht als Bacteriencylinder bezeichnen kantr. Dann zerfallen die Cylinder völlig, 'die Bacterien werden wieder frei, und an Stelle der Cylinder bleiben nur Reste von schwerlöslichem Detritus übrig.

Dieser Process braucht aber selbst bei höherer Zimmertemperatur imner längere Zeit, zumal wenn die Urine ursprïnglich stark samer sind. Da die Differenzen in der Cyliniderzahl aber schon in den frisch entleerten Urinen vorhanden sind, welche noch keinerlei Fänhrissorganismen enthielten, so kam diese Erklärung hier gleich-

1) C. L. Rovida, Arch. per le sciente mediche.: Torino; Bd. I, 1877. falls nicht \%utreffend sein. Es ist ja allerdings zuzugeben, dass bei der gewöhnlich geïbten Art der Untersuchung, bei der man den Urin 24 Stunden lang bei Zimmertemperatur sedimentireu lässt recht wohl schion eine Lösung der Cylinder sich bemerlklich machen kaun, zumal weun ter Urin nur eine geringe Acidität besass. Fï die Differenzen in frischen Urin bleibt uns aber nach dem $\mathrm{E}_{\mathrm{r}}$ örterten nurr noch die Möglichkeit einer Auflösung der Cylinder lurch ungeformte Fermente ïbrig.

Vou den Fermenten, welche innerhalb des Körpers Eiweiss oder eiweissälmliche Substanzen \%a lösen. vermögen, kommen überlaupt nur zwei, das Pepsin und Trypsin, für unsere Frage in Betracht.

Trypsin kommt im normalen Harn nicht vor, hiervon habe ich mich durch selır zaliheiche Versuche überzengt, ind mit dieser Annahme stimnen auch die neueren Arbeiten ïber diesen Punkt wohl durchweg überein. Ehensowenig hat sich bisher in nephritisclen Harnen Trypsin nachweisen lassen.

Es bleibt also für eine solche Fermentwirkung uur das Pepsin übrig. Das Pepsin ist nun nach den sehr zahlreichen, auf diesen Punkt gerichteten Untersuchungen ein fast regelmässiger Bestandtheil des Urins. Seine Menge unterliegt täglich gewissen, von ,der Nahrungsaufuahme und Magenverdaumg ablängigen Schwankungen und erreicht zuweilen ganz beträchtliche Grössen. Da zudem neuerdings von Stadelmann ${ }^{1}$ ) mit voller Bestimmtheit nachgewiesen worden ist, dass der in saurer Lösnng proteolytisch wirkende F'ermentkörper des Urins wirklich Pepsin ist, so könoen wir dieses Factum als durchaus gesichert annehmen, und da keiner der Autoren ein Fehlen des Pepsins bei Nephritis oder Albnminurie aus sonstigen Ursachen zu constatiren vermochte, sind wir in der That berechtigt zu fragen, ob das Verschwinden der Cylinder im Urin auf einer Lösung durch Pepsin beruht.

Die Frage wird allerdings nur dann für entschieden geltel können, wenn erstens das Pepsin schon im Urin selbst \# wirken vermag, zweitens wenn die Cylinder aus einer Substanz bestehen, die der Pepsinverdaumng zugänglich ist, und drittens, wem sich eine Parallele auffinden lïsst zwischen den Mengen des Pepsins und der Cylinder, und zwar in der Art, dass ein Ansteigen der Pepsinmenget stets you einem entsprechenden Abfall in der Zahl der Cylinder begleitet ist und urngekehrt.

Der Urin selbst könnte auf doppelte Weise störend auf die Pepsinverdauung einwirken. Einmal ist es eine bekanute Thatsache, dass ein höherer Gehalt der Verdauungsflüssigkeit an Sal'zen die peptische Proteolyse stark hemmen und selbst sistiren kann. Zweitens wirkt das Pepsin am besten, wenn die saure Reaction seiner Lösung durch freie Säuren, am vortheilhaftestev mineralisclic Säuren, bedingt ist. Schwächer schon ist die Wirkung bei organischen Säuren.. Die saure Reaction des Urins beruht nun mur zum geringen 'Theil auf freien organischen Säuren, vorwiegend auf cler Gegenwart saurer Salye. Es ist daher die Frage, ob auch diese Art saurer Reaction ausreichend für eine Pepsinwirkung ist.

Am einfachsten entscheidet man beide Fragen durch direkte Verdauungsversuche mit dem Urin selbst. Als ich zu anderen Zwecken bei verschiedenen Personen stüudliche Pepsinbestiın nnngen des Urins ausfïhrte, ist mir die Wirkung des Pepsins in saurem Urin oft in recht storender Weise entgegengetreten. Sucht man das Pepsin in der Art nachzuweisen, class man es ans den sterilisirten Urinen $\left.{ }^{2}\right)$ durch Aufsaugen mittels Fibrin isolirt, so begegnet es einem hïufig, dass in manchen Urinportionen das Fibrin schon nach wenigen Stunden vollständig gelöst nud verschwunden ist, während es iit anderen Portionen unter sonst gleichen Bedingungen tagelang völlig unverändert bleibt. Eine Lösung des Fibrins durch Fäulniss ist bei der Sterilisirung der Urine natürlich völlig ansgeschlossen, ebenso wentig können aber anch ungeforrnte, schon vorher von den Mikroorganismen abgespaltene Fermente diese gewaltige Wirkung veranlasst liaben; denn solche Fermente können, wenn man überlaaupt die Möglichkeit ihrer Existenz zugeben will, in unseren Urinen, die stets völlig frisch verwandt" wurden, duichaus noch nicht, oder höchtstens in minimalen Spuren vorhanden gewesen sein, und wir sind daher in der That berechtigt, anzunehmen dass anch in dent relativ sal\%reichen Urin, mit seiner vorwiegend durch saure Salze bedingten Acidität, eine Pepsinwirkung möglich ist und eintritt.

Der zweite Factor, welcher für uns in Betracht kommt, ist die chemische Beschaffenheit der Harncylinder: Bekanntlich ist diese Frage \%ur Zeit noch durchaus nicht endgültig entschieden dem während manche Autoren die Cylinder einfach für geronnenes Eiweiss, das aus dem Blutserum stanmt, erklären, bestreiten andere überhaupt, dass die Snbstanz der Cylinder ein Eiweisskörper sei, und wollen sie höchstens für einen eiweissähnlichen, alburninoiden

1) E. Stadelmann, Ueber Fermente im normalen Harn. Zeitschr. f. Biologie, 13. 24 , p. 226 und Bd. 25

E. Sehrwald, Ueber die Sterilisirung und den Nachweis ungefornter 
Stoff gelten lassen. Jedenfalls mnss man bei der Chemie der Cylinder berïcksichtigen, lass wohl nicht alle Arten von Cylindern eine gleiche Genese nnd Znsammensetznng besitzen. Man hat vorwiegend drei Entstehnngsarten fïr die Cylinder nuterseheilen wollen. Erstlich vernag das Eiveiss, welches bei der Albnninurie im Glomerulus austritt, inierhalb der Harncauälchen zn hyalinen Massen zu gerinuen, entweder unter der Mitwirknug gleichzeitig ansgetretener ind zerfallender Lenkocyten, oder nnter Beiliïlfe zerfallender Nierenepithelien oder endlich, wie Ribbert') nachgewiesen hat, cinfach durch den Einflnss gewisser normaler Harnbestand theile. Ribbert fand bekanntlich, dass das im Glomernlns ansgeschiedene Eiweiss schon infolge der saneren Reaction des Harns hyalin zu gerinnen vermag, dasselbe Resnltat erhielt er aber auch durch verdiinnte, reine Harnsäurelösnngen, durch verdiinnte Salzsäure, Phosphorsänre, nnd endlich anch in nicht sanrer Lösung direkt durch die Einwirkung des Harnstoffs.

Während hier also das Material zur Bildnng der hyalinen $\mathrm{Cy}$ linder vom Glomernhus her geliefert wird, leiten andere, wie Axel Key, $\left.{ }^{3}\right)$ Oertel, Rovida") n. s. w. die Entstehnng dieser Cylinder von einem Secretionsprocess der Canalepithelien ab. Schon in der Norm sollen diese Epithelien feine Tröpfehen hyaliner Substanz enthalten nnd danernd in geringer fïr die Uriumntersuchnng nicht nachweisbarer Menge ansscheiden. Bei krankhaften Processen der Niere wird nnu die Bildnng dieser hyalinen Substanz gesteigert, sie ragt in Form von Tropfen ans den Epithelien herans, die Tropfen ergiessen sich in das Lnmen der Harucanälchen nnd verschmelzen hier zn cylindrischen Massen.

Eine dritte Erklärnng endlich lässt die erkrankten Epithelien der Harncanälchen selbst znr Bildnng eler Cylinder verwandt nnd aufgebraucht werden.

Es ist an dieser Stelle nicht meine Anfgabe, zn untersuchen, wie weit die einzelne Hypothese richtig und berechtigt ist. Eins haben alle diese Hypothesen gemein. Sie fülıren alle die Entstehnng der Cylinder auf echte Eiweisskörper znrïck, entweder anf die gelösten Eiweisskörper des ausgetretenen Bintserums oder anf clie organisirten Eiweisskörper in Zellprotoplasma der Niere. Wenn nıı cliese Eiweisskörper auch bei der Umwandlung zn Cylinderı gewisse physikalische nnd wohl auch chemische Veräıdernngen erfahren, so bleibt ihre Substanz doch nach der übereinstimmenden Ansicht der Antoren dem Eiwciss sehr nahe verwandt, und wir clïrfen mit einigem Recht voranssetzen, dass diese Substanz in ganı ähnlicher Weise wie die echten Eiweisskörper der Pepsinverdannog nnterliegen mag.

Um mir über diesen Punkt Gewissheit zn versehaffen, habe ich Urine, die sehr zahlreiche Cylinder der verschiedensten Art enthielten, der Pepsinverdaunng nnterworfen. Die Urine wurden mit Salzsäure, bis zu $0,1 \%$, nud Pepsinglycerin versetzt. Ansserdem wurde durch reichlichen Znsatz von Chloroform die Fünlniss ansgeschlossen und die Lösung nnter Watteverschlnss 24 Stunden in den Brïtofen gesetzt. Eine etwaige Auflösung der Cylinder konnte dann nnr durch die Pepsinwirknng bedingt sein. Denn die $0,1 \%$ ige Salzsäurelösung allein konıte daran nicht Schnld sein, da nach Ribbert's Untersnchnngen, wie schon erwähnt, das ans dem Glomernlns ausgeschiedene Eiweiss ja gerade dnrch verdiinnte Salzsänre als byaline Masse ansgefällt wird.

In der That waren nun nach 24 Stnnden alle Cylinder verschwnnden, während das Gesammtsediment des Urins kaım an Menge verloren hatte. Das Sediment bestand jetzt fast ansschliesslich aus Köruchenhanfen, die mur vereinzelt noch die Anordnnng beibehalten hatten, die sie in den Cylindern gehabt, meist waren sie mit anderen Häufchen zn nnregelmässigen Fignren confluirt. Ansserdem waren zahlreiche Zellkerne im Sediment noch erhalten. Hyaline, amyloide, Körnchen- und Epithelcylinder, soweit diese einc hyaline Grnudlage besitzen, waren durchans nicht mehr nachweisbar. Nur eine Art von Cylindern war in änsserst vereinzelten kleinen Trümmern noch erhalten, nämlich in toto und sehr grobkörnig grannlirte Cylinder von anffallend dnnkler Färbnng.

Es darf also als gesichert gelten, dass alle hyalinen, alle mit hyaliner Grnndlage versehenen nnd ferner die direkt ans diesen hervorgehenden Formen derCylinder dnrch die Pepsinverdannng anfgelöst werden. Hierdurch wird die nahe Verwandtschaft der hyalinen Substanz der Cylinder zn den Eiweisskörpern gleichfalls bewiesen. Aus welchem Material hingegen die spärlichen, stark grannlirten und niclit im Lanfe von 24 Stunden gelösten Cylinder bestehen, nunss weiterer Untersnchnng vorbehalten bleiben.

Nachdem also nachgewiesen ist, dass das Pepsin in sanrem Urin zn wirken vermag, nud dass zweitens die Cylinder mit sehr p. 305 .

) H. Ribbert, Centralbl. f. d. medicin. Wissensch. 1581, Bd. 19,

${ }^{2}$ ) Axel Key, Om de s. K. Tubalara fgjutringarnas etc. Stockholm 1863 .

3) Rovida, Nöleschott. Untersuchungen z. Naturlehre, Bd, XI. geringer Ansnahune durch das Pepsin verdaut werclen können, bleibt drittens nnr noch ïbrig, festznsteHen, ob in der That die Schwanknngen in dér Zahl der Cylinder anf entsprechende Differenzen im Pepsingehalt des Urins sich zurn̈ckfiiluren lassen.

Ich habe zn den Zweck nach den bekannten Methoden die Pepsinmengen in den Uriuen bestimmt, in denen ich auch die Zah der Cylinder untersucht hatte. Ans den beiden angefïgten Cnrven sieht man mun, dass in der That eine enge Beziehnug zwischen den Mengen der Cylinder nud des Pepsins besteht. Sobald die Pepsinmengen ansteigen nnd also eine ansgiebigere Verdanung der Cylinder möglich wird, sinkt chie Zahl der Cylinder ab; sobald der Gehalt an Pepsin heruntergeht, nimmt umgekehrt der an Cylindern wieder zu.' Dies Verhalten prägt sich in beiden Cnrven gleich dentlich aus.

Es braneht wohl nicht daran erimnert zn werden, dass die verdanende Kraft eines Urins nicht bloss von der Nenge seines Pepsins, sondern zweitens anch noch von dem Grad seiner Acidität abhängt. Die Pepsincurven geben nnu die peptische Wirkung der Urine all bei der betreffenden Acidität, welche die einzelnen Urinportionen besassen. Sie sollen also gewissermaassen die Resnltirenden der beiden Factoren; Sänre nnd Pepsin, znr Anschannng bringell, da unr diese ein Ansdrnck fïr die verdanende Kraft, auf die es hier allein ankommt, sein können.

Sind diese Thatsachen richtig, so nin̈ssen sich noch weitere Folgernngen hierans ergeben. Vor allem ist eiulenchtend, dass in einem cylinderhaltigen Urin die Zahl der Cylinder anch bei $\Lambda$ us. schlnss der Fänhniss immer mehr abıehmen mnss, je länger der Urin steht. Diese Abnahme mnss nm so bedentender sein, je höher die peptische Kraft des Urius und je höher die herrschende Temperatnr ist. Setzt man gleiche Mengen eines solchen Urins nach Chloroformznsatz 24 Stnnden in die Kälte und die zweite Portion ebenso lange in den Brïtofen, so zeigt der kalt gehaltene Urin nach dieser Zeit nnr eine geringe Abnalıme seiner Cylinder, während der Urin im Brïtofen viel cylinderärmer geworden ist, zngleich sind aber die noch erhaltenen Cylinder viel blasser und zarter als znvor. Setzt nuan gar der Urinportion in Brïtofen noch künstlich Pepsin $\mathrm{zn}$, so ist die Verminderung der Cylinder noch weitans hochgradiger nud ihr Anssehen noch bedentend schemenhafter.

Für die Praxis ergiebt sich hierans ohne weiteres zweierlei. Will man in einem Urin mit möglichster Sicherheit etwaige Cylincler nachweisen, so empfiehlt es sich, den Urin nicht \%n lange nnd vor allem nicht bei zn hoher Zimmertenperatur sedimentiren on lassen, da sonst alle vorhandenen Cylinder wieder völlig verschwinden können. Zweitens aber muss es äusserst rathsam erscheinen, nicht anch noch der Fänlniss Gelegenheit zn geben, an diesem Zerstörnngswerk mitznarbeiten. Da anch schon im sanren Urin eine reichliche Entwickelnng von Mikroorganisnen auftritt, erscheint es rathsam, säıntliche Urine mit Chloroform im Ueberschnss zn versetzen, event. sie nit dem Chloroform leicht durchznschütteln. Die meisten Cylinder sind von so fester Strnetnr, dass sie eill solches Schïttcln git ertragen, ohne zn zerreissen und zn zerstïckeln.

Da die verdaneilde Wirkıng des Pepsins am kräftigsten bei Körpertemperatur erfolgt, so mïssen die Cylinder in einer Urinportion nm so ansgiebiger verschwinden, je länger dieselbe innerhalb der Blase verweilt. Je seltener daher Urin gelassen wird, nm so núsicherer wird bei geringer Zahl von Cylindern deren Nachweis werden 'müssen. Dass thatsächlieh bei längerem Zurïckhalten des Urins in der Blase eine Vermindernug der Cylinder wieder eintreten mnss, geht anch ans unseren Cnrven hervor. Bei der zweiten Cnrve wnrdeı die Urinmengen möglichst selten entleert, nnd die absolnt grösseren Harnmengen hätten daber anch absolnt grössere Mengen an Cylindern anfweisen müssen. Statt dessen sind diese Mengen eher kleiner, höchstens ebenso gross als in clen stïndlich entleerten Portionen. Mall wird deshalb bei schwierigem Nachweis von Cylindein nicht nur den Urin möglichst frisch nutersnchen, sonderi auch clafür Sorge tragen müssen, dass er nur möglichst knr\%e Zeit in der Blase verweilt.

Die neisten Arbeiten ïber das Pepsin im Urin sprechen diesem Stoff jede Bedentnng ab. Es ist ein Answurfsstoff, der bei der Verdanung seine Schnldigkeit gethan hat und den der Körper unn wegwirft, wie so viele andere. Dass dieser Körper aber trotzdem noch einmal Spnren seiner Macht zeigen kann, indem er nnsere Diagnose erschwert, habe ich gezeigt. Es ist die Frage, ob dieser Stoff, sobald er in den Urin gelangt ist, nicht anch znweilen noch iın gïnstigen Simne für den Organismns zn wirken vermag, und das ist nicht ohne weiteres in Abrede zn stellen.

Wie die Cylinder innerhalb der Blase vom Urin verdant werden, so dïrfen wir wohl anch voranssetzen, dass das Pepsin anch schon seine Arrosion an den Cylindern beginat, so lange diese noch in den Harncanälchen festsitzen. Znmal bei der Nephritis müssen wir wohl annehmen, dass die Cylinder in den Harncallälchen anfangs mit dem Epithel riemlich fest verklebt sind, einerlei ob die Cylinder 
erst durch Ausfällen von im Urin gelöstem Eiweiss innerhalb der Harncanälchen oder direkt durch Umwandlung der Canalepithelien entstehen. Für eine solche Verklebung spricht vor allem das Festhaften von Nierenepithelien an den Cylindern. Findet nun eine solche Verklebung zwischen den Wandungen der Canälchen und den Cylindern wirklich statt, so muss anfangs eine volle Verlegung dieser Canälchen durch die Cylinder eintreten, die um so länger andauert, je schwerer der Harnstrom die Gerinnsel loszulösen vermag. Ein solcher Verschluss der abfïhrenden Harnwege erhöht nun unbedingt den Gegendruck in denselben und vermindert damit die Secretion des zugehörigen Nierenabschnittes. ${ }^{1}$ ) Cohnheim ${ }^{2}$ ) meint zwar, dass Zerfallsmassen und Cylinder innerhalb der Harncanälchen höchstens ein minimales Hinderniss für den Abfluss des Harnes abgeben und mit Leichtigkeit vom Harnstrom durchbrochen werden. Das ist aber nur dann richtig, wenn diese obturirenden Massen nicht fest mit den Wändelı der Canälchen verklebt sind.

Da bei Festhaften der Cylinder in den Canälchen zugleich auch der Harnstrom vermindert werden muss, der dieselben durehfliesst, so würden die Cylinder um so schwerer wieder aus der Niere fortgeführt werden können, wenn nicht ein neuer Factor die Lockerung und Loslösung der Cylinder befördern würde, und einen solchen Factor diirfen wir in dem Pepsingehalt des Urins erblicken. Unterstïtzt wird eine solche Lösung der Cylinder noch durch die Fähigkeit der geronnenen Eiweisskörper, das Pepsin auf sich niederzuschlagen. Früher war diese Fähigkeit nur vom Blutfibrin bekannt, Fick ${ }^{3}$ ) hat nun gezeigt, dass sie allen geronnenen Kiweisskörpern zukommt. Wir dürfen sie daher wohl auch für die Substanz der Cylinder mit annehmen. Nach den Angaben von $\mathrm{Zie} \mathrm{gle} \mathbf{r}^{4}$ ) scheinen übrigens die Cylinder in der Niere selbst schon wieder völlig aufgelöst werden zu können. Er hebt hervor, dass die Cylinder häufig in der Niere längere Zeit liegen bleiben, wachsartig oder wieder völlig aufgelöst werden. Die Möglichkeit einer solchen Wiederauflösung würde nach dem Erörterten höchstens durch den Pepsingehalt des Urins eine Erklärung finden.

Aber nicht bloss die Massen der Cylinder, sondern auch alle anderen todten Eiweissstoffe, die in den Canälchen einer kranken Niere siel aufhäufen, wie abgestorbene Epithelien u. s. w., werden unter der Beihülfe des Pepsins leichter aus der Niere entfernt. Die peptische Verdauung erleichtert in der Niere die Demarcation des gesunden Gewebes gegen das dem Untergang verfallene. Das gesunde Gewebe selbst wird durch diese Verdauung nicht angegriffen, ebenso wenig wie die lebende Magenschleimhaut, und wie ich von dieser nachgewiesen habe ${ }^{5}$ ), dass nicht die Alkalescenz des Blutes das Schutzmittel bildet, sondern dass diese iin den Eigenschaften der lebenden Zelle gegeben ist, so müssen wr das Gleiche auch fïl die Niere annehmen.

Fasse ich die Hauptpunkte der Untersuchnung nochmals in Kürze znsammen, so glaube ich folgendes gezeigt zu haben:

1. Bei typischer Nephritis gelingt es an manchen Tagen nicht, Cylinder im Urin nachzuweisen, obgleich dieselben an anderen Tagen sich reichlich finden, und obgleich der Eiweissgehalt des Urins gleichzeitig keine wesentliche Schwankung aufweist.

2. Die stündlichen Harnportionen eines Tages zeigen oft sehr bedeutende Differenzen in der Menge der Cylinder bei anıähernd gleichem Eiweissgehalt.

3. Die cylinderarmen Portionen enthalten dafür grössere Mengen von Körnchenhaufen und freien Kernen. Die etwa noch vorhandenen Cylinder sind viel blasser, zarter und schemenhafter, als bei reichlichem Cylindergehalt.

4. Auf eine abwechselnde Thätigkeit bald mehr gesunder, bald stärker erkrankter Partieen der Nieren lassen sich diese Differenzen nicht zurückführen, da die cylinderarmen Portionen dann auch weniger Eiweiss und mehr Harnwasser enthalten müssten, als die anderen, was nicht der Fall ist.

5. Das reichlichere Vorkommen von Detritusmassen bei geringer Cylinderzahl und die zartere Beschaffenheit letzterer deutet darauf hin, doss eine Wiederauflösung der Cylinder im Urin stattgefunden hat.

6 . Fine einfache chemische Auflösung der Cylinder kann daran nicht Schuld sein, da die normalen Bestandtheile des sauren Urins gerade umgekehrt die Eiweisskörper im Urin ausfällen.

Ebenso wenig können Fäulnissorganismen die Lösung bewirkt haben, da auch die völlig frischen, bacterienfreien Urine die Differenz schon zeigen.

7. Die Lösung der Cylinder kommt durch den Pepsingehalt

1) E. Sehrwald, Die Bedeutung des Nervensystems f. d. Niere. Jena, (x. Fischer 1887 , p. 76.

2) Cohnheim, Vorlesung. ïber allo. Patholog. 18s2, II, 393.

3) Fick, Ueber die Anziehung des Pepsins durch Eiweisskörper. Sitz.Ber. d. physikal.-med. Gesellsch. zu Würzburg 1889, No. 2, p. 23.

4) E. Zi egler, Lehrb. d. speciell. path. Anat. II, I885, p. 425.

5) E. Seh rwa ld, Was verhindert die Selbstverdauung d. lebenden Magens? München. med. Wochenschr. 1888, No. 44,45 . der saueren Urine zustande. Das Pepsin vermag im sauren Urine proteolytisch zu wirken. Die Pepsinverdaunng ist imstande, die Cylinder mit geringer Ausnahme aufzulösen. Ein Anstieg der Pepsinwirkung im Urin ist begleitet von einer Abnahme in der Cylinderzahl, wie es diese Annahme erfordert.

8. Je länger man den Urin in der Blase verweilen oder bei höherer Zimmertemperatur sedimentiren lässt, um so mehr nimmt die Zahl der Cylinder ab.

9. Praktisch empfiehlt sich daher, den Urin nur kurz und bei niederer Temperatur sedimentiren zu lassen, um die Fäulniss auszuschliessen, ihn dabei mit Chloroform im Ueberschuss zu versetzen, und Urinportionen $\%$ benutzen, die nur möglichst kurze Zeit in der Blase verweilt haben.

10. Diese peptische Verdauung kann, wie in der Blase, so auch schon in der Niere selbst beginnen und dadurch in den Harncanälchen festhaftende Cylinder lockern und ihre Ausscheidung fördern. 